\title{
Efecto del juego vs. el role-playing en la implicación de los participantes del Programa SALUDIVERSEX ${ }^{1}$
}

\section{María Dolores Gil-Llario}

Departamento de Psicología Evolutiva y de la Educación, Universitat de València, Valencia, España

\section{Dolores.Gil@uv.es}

\section{Verónica Estruch-García}

Departamento de Psicología Evolutiva y de la Educación, Universitat de València, Valencia, España

veronicaestruch11@gmail.com

\section{Olga Fernández-García}

Departamento de Psicología Evolutiva y de la Educación, Universitat de València, Valencia, España

Olga.Fernandez-Garcia@uv.es

\section{RESUMEN}

El programa de educación afectivo sexual SALUDIVERSEX para personas con diversidad funcional intelectual (DFI) sienta sus bases sobre la importancia de la implicación activa de los participantes en las sesiones dirigidas a favorecer el aprendizaje de conocimientos, habilidades y actitudes saludables. Por ello, en las sesiones dedicadas a transmitir conocimientos sobre sexo/sexualidad, higiene sexual, prácticas sexuales y sexo seguro, así como, en las que se trabajan actitudes hacia la diversidad sexual y las relaciones de pareja se prioriza más el juego. Por otra parte, en las sesiones dedicadas a trabajar habilidades interpersonales se emplea fundamentalmente el role playing. El objetivo del presente trabajo es observar el grado de implicación de 29 usuarios/as (el 55\% son hombres y el $45 \%$ son mujeres) que participaron en el programa SALUDIVERSEX en función del tipo de metodología empleada en las diferentes sesiones. La edad de los participantes está entre los 25 y 65 años ( $\mathrm{x}: 39,775$; D.T.: 9,03). El grado de implicación fue valorado por los/as profesionales en una escala de 0 a 2 . Los resultados sugieren que ambas metodologías son útiles observando una alta implicación tanto en las sesiones de habilidades $(1,224)$ como en las de actitudes $(1,216)$. Los peores resultados hallados en cuanto a implicación en las sesiones de conocimientos $(0,995)$ podría deberse a que las temáticas trabajadas abordan diferentes aspectos de la sexualidad de forma más explícita, lo que contribuye a que se muestren más retraídos al no estar habituados a comentar aspectos relacionados con la sexualidad en público. Estos hallazgos nos llevan a concluir que, es necesario que los/las profesionales formados/as durante el programa apliquen lo

\footnotetext{
${ }^{1}$ Esta investigación ha sido realizada gracias a un proyecto de investigación financiado por el Programa Estatal de I+D+i de la Convocatoria 2018 Orientada a los Retos de la Sociedad del Ministerio de Ciencia. Innovación y Universidades (Proyecto RTI2018-095538-B-100).
} 
aprendido, integrando la educación sexual como una actividad más en sus respectivos centros ocupacionales a fin de normalizar la sexualidad en este colectivo.

Palabras clave: Programa SALUDIVERSEX; discapacidad intelectual; motivación, role playing; juego.

\begin{abstract}
The program of sexual education for people with intellectual disabilities SALUDIVERSEX is based on the relevance of active implication of the participants in the sessions to promote healthy knowledge, skills and attitudes. Therefore, in the sessions dedicated to conveying knowledge about sex/sexuality, sexual hygiene, sexual practices and safe sex, as well as, in the sessions dedicate to work attitudes for the sexual diversity and relationships are prioritized more the play. On the other hand, in the sessions dedicated to work interpersonal skills are mainly employed role-playing. The objective this work is to observe the degree of involvement of 29 participants of SALUDIVERSEX' Program (55\% are men and $45 \%$ are woman) depending on the type of methodology used in the different sessions. The participants are between 25 and 60 years old. The professionals valued the degree of involvement of participants on a scale of 0 to 2 . The results suggest that both methodologies are useful as users had a high involvement in both skill and attitude sessions. The worst results presented in knowledge sessions could be explained by the topics worked on in these sessions, as sexuality is more explicitly addressed, which could contribute to participants being more withdrawn by not being accustomed to commenting on aspects related to sexuality in public. These findings lead us to conclude that, it is necessary for professionals trained during the program to apply what they have learned, integrating sex education as one more activity in their respective occupational centers in order to standardize sexuality in this population.
\end{abstract}

Key Words: SALUDIVERSEX' Program; intellectual disabilities; motivation; role-playing; play.

\title{
INTRODUCCIÓN
}

La educación sexual es una herramienta fundamental para que las personas desarrollen actitudes y comportamientos saludables y satisfactorios respecto a su sexualidad (Navarro, 2012; Verdugo et al., 2002). Aunque actualmente se reconoce la importancia de promover un desarrollo sexual saludable en la población general, existe aún cierta controversia cuando se aborda la sexualidad de la población con diversidad funcional intelectual (DFI) (Gil-Llario, Ballester-Arnal, et al., 2019; Roden et al., 2020). La investigación en este campo ha permitido conocer algunas de las limitaciones que presentan las personas con DFI que dificultan la vivencia de la sexualidad de forma satisfactoria y placentera, y que se derivan de las condiciones asociadas a sus necesidades de apopo. Por una parte, las personas con DFI tienen bajos conocimientos sobre los métodos de prevención de embarazos y enfermedades de transmisión sexual (Aceldo et al., 2006), lo que aumenta el riesgo de exponerse a conductas sexuales de riesgo. Por otra parte, este colectivo presenta una alta vulnerabilidad a sufrir abusos 
sexuales. En este sentido, Gil-Llario, Morell-Mengual, et al. (2019) hallaron que los participantes de su estudio presentaban cierta tendencia a infravalorar el riesgo a sufrir abusos sexuales por parte de las personas de su entorno y tenían dificultades para comprender el concepto de abuso sexual, lo que aumenta su vulnerabilidad a sufrir experiencias negativas de esta índole (Giménez-García et al., 2017). Así mismo, la literatura ha destacado las pocas habilidades sociosexuales que presentan, un tema que preocupa bastante a este colectivo (Gil-Llario, Ballester-Arnal, et al., 2019), ya que perciben que tienen pocas posibilidades de encontrar pareja (Whittle \& Butler, 2018). A esto cabe sumar las creencias erróneas (Navarro, 2012) y expectativas poco realistas que tienen sobre las relaciones románticas promovidas, en parte, por la exposición a los modelos sociales presentados en los medios de comunicación (Navarro et al., 2010).

Actualmente son cada vez más las personas con DFI que reciben educación sexual, pero parece que esta formación no siempre ha alcanzado a cubrir las expectativas y necesidades de los participantes, dado que estos continúan expresando su deseo de recibir más información sobre sexualidad (Díaz-Rodríguez et al., 2014). Entre las críticas que han recibido este tipo de intervenciones encontramos algunas que hacen referencia al enfoque desde el que abordan la sexualidad, en muchos casos limitado a la prevención (Cosentino, et al., 2010), lo que sin duda es esencial, pero insuficiente para dar una respuesta integral a las necesidades sexuales de este colectivo. Por su parte, Schaalma et al. (2004) destacaron que muchos de estos programas se limitan a mejorar el conocimiento de los participantes, no atendiendo al desarrollo de habilidades y actitudes positivas, factores que resultan esenciales para que se produzcan los cambios conductuales deseados. En la misma línea, Jahoda \& Pownall (2014) destacaron la falta de atención de las actitudes hacia la sexualidad y el placer sexual del colectivo con DFI. Por todo ello, el programa de educación afectivo sexual para adultos con diversidad funcional intelectual SALUDIVERSEX (Gil-Llario et al., 2019) promueve la salud sexual accionando sobre los conocimientos, habilidades y actitudes sobre sexualidad, a través de 16 sesiones cuyas dinámicas favorecen la implicación activa de los participantes. Estos autores recomiendan que sean los profesionales que trabajan habitualmente con los/as usuarios/as, quienes lleven a cabo la implementación del programa dada la relación de confianza que ya tienen establecida.

Sin lugar a duda, estas iniciativas constituyen un gran paso en la integración de las personas con DFI en la sociedad y reflejan la preocupación de los investigadores por mejorar las condiciones de vida de este colectivo. No obstante, una de las críticas más referenciadas es la escasa evidencia empírica sobre su eficacia (Kellam \& Langevin, 2003; Whitehouse \& McCabe, 1997). Así mismo, existe un gran desconocimiento sobre el efecto de las metodologías empleadas en estos programas sobre el aprendizaje. La relación positiva entre la motivación y el aprendizaje hace que sea esencial conocer aquellas estrategias que promueven de forma efectiva la implicación de los/as participantes, especialmente teniendo en cuenta que en este colectivo, el simple hecho de transmitir información no resultará el efecto deseado (Schaafsma et al., 2013).

Por ello, con la finalidad de llenar el vacío existente sobre el efecto de la metodología de enseñanza en la implicación de los/as participantes de los programas de educación afectivo-sexual, el presente trabajo se plantea el objetivo de estimar el grado de implicación durante el programa SALUDIVERSEX de los/as beneficiarios/as del mismo, en función del tipo de metodología empleada en las diferentes sesiones (role playing vs. juego). 


\section{MÉTODO}

\section{Participantes}

La muestra empleada en este estudio está conformada por 29 usuarios/as procedentes de 3 centros ocupacionales de la provincia de Castellón (Comunidad Valenciana) que participaron en el Programa SALUDIVERSEX. El 55\% son hombres y el $45 \%$ son mujeres, cuya edad está entre los 25 y 65 años $(\bar{x}=39,775 ; D T=9,03)$. Todos los participantes tienen un diagnóstico de discapacidad intelectual con un CI menor a 80.

\section{Procedimiento.}

A partir de la demanda realizada al grupo de investigación SALUSEX por parte del Instituto Valenciano de Atención Socio-sanitaria que atiende a personas con DFI, se llevó a cabo la formación sobre la implementación del programa SALUDIVERSEX a los profesionales de 3 de los centros ocupacionales que forman parte de esta red de servicios en Castellón. Una vez los/as directores/as de los diferentes recursos y los padres y las madres de los/as usuarios/as dieron su consentimiento para participar en el programa, se inició la implementación de este. Este programa está compuesto por 16 sesiones de dos horas de duración y fue aplicado por dos profesionales de cada centro ocupacional, formados a tal efecto, con una cadencia semanal. Cada centro ocupacional seleccionó entre 8 y 10 usuarios/as para participar en el programa, siguiendo los siguientes criterios: ser mayor de 18 años y tener un diagnóstico de discapacidad intelectual leve o moderada. Así mismo, se instruyó a los/las profesionales para que evaluaran la implicación del/de la usuario/a en cada una de las 16 sesiones que componen este programa, en función del número de preguntas pertinentes que realizaban, empleando una escala de 0 a 2, dónde 0 hace referencia a que el/la usuario/a realiza pocas preguntas pertinentes y 2 a que interviene realizando muchas preguntas pertinentes.

\section{Análisis de datos.}

Una vez recogidos los informes sobre la valoración de los/as profesionales, se extrajo el promedio de las preguntas pertinentes que había realizado cada grupo de participantes, según el contenido de las sesiones y la metodología de enseñanza predominante. Por tanto, se analiza el promedio de preguntas pertinentes de los/as usuarios/as en tres tipos de sesión: las sesiones que fundamentalmente se dedican a fomentar el desarrollo de habilidades sociosexuales (comunicación, prevención de abusos sexuales, habilidades para iniciar, mantener y finalizar relaciones afectivas y asertividad sexual), en las que se priorizan dinámicas de role playing como método de enseñanza; las sesiones que principalmente se dedican a mejorar los conocimientos (sexualidad, higiene sexual, intimidad, prácticas sexuales, métodos anticonceptivos e ITS), en las que se emplean fundamentalmente dinámicas de juego; y las sesiones dedicadas a promocionar actitudes positivas hacia la sexualidad (autoestima, diversidad sexual y ética de la pareja), en las cuales también se prioriza el juego como método de aprendizaje.

\section{RESULTADOS}

En relación a la implicación de los/las participantes de este estudio, como se puede observar en la gráfica 1, el promedio de preguntas pertinentes más alto se da en las 
sesiones dedicadas al desarrollo de habilidades en las que se prioriza el uso del role playing $(\mathrm{X}=1,224)$, ya que los/las participantes realizan entre bastantes y muchas preguntas pertinentes. En segundo lugar, observamos que el grado de participación es ligeramente menor en las sesiones centradas en las actitudes $(X=1,216)$, en las que se emplea principalmente el juego, aunque en este caso los usuarios y las usuarias también se sitúan entre bastantes y muchas preguntas pertinentes. En último lugar, los/las participantes presentan un menor grado de implicación en las sesiones destinadas a transmitir conocimientos en las cuales también se prioriza el juego para trabajar conceptos $(\mathrm{X}=0,995)$, siendo en este caso el promedio de preguntas pertinentes ligeramente inferior al nivel bastantes preguntas pertinentes.

\section{Gráfica 1.}

Grado de implicación en función del contenido de la sesión: promedio de preguntas pertinentes.

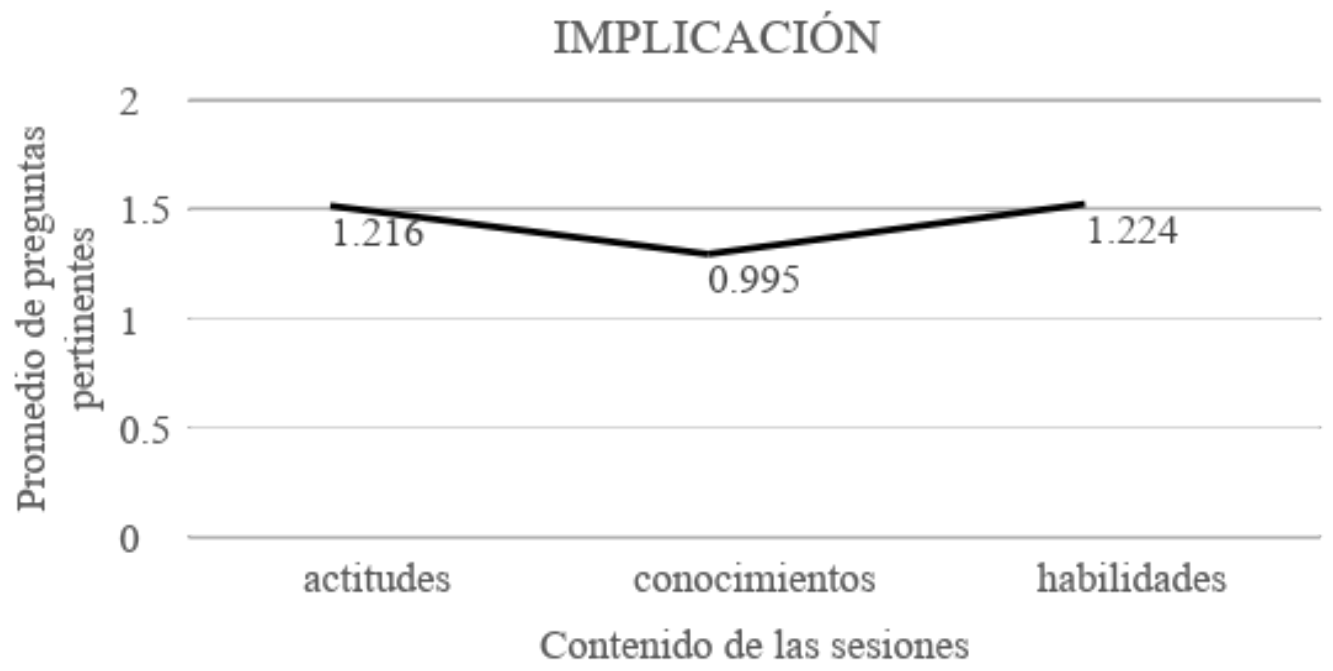

\section{DISCUSIÓN}

El objetivo de este trabajo fue observar el grado de implicación de los/las usuarios/as que participaron en el Programa SALUDIVERSEX, en función de la metodología de enseñanza empleada en las diferentes sesiones (role-playing vs. juego). Según los resultados obtenidos en el presente estudio ambas metodologías (role playing y juego) son útiles para fomentar la participación de los/las usuarios/as, dado que se observa una alta implicación tanto en las sesiones de habilidades como en las de actitudes. Por un lado, la formulación de más preguntas pertinentes por parte de los/as participantes en las sesiones centradas en el desarrollo de habilidades podría explicarse por las temáticas tratadas en las mismas, dado que la interacción con iguales con fines sexuales es algo que preocupa bastante a este colectivo (Whittle \& Butler, 2018). Por otra parte, dado que el efecto del juego sobre la motivación de los/as participantes en las sesiones actitudinales es bueno, los peores resultados en las sesiones de conocimientos podrían deberse a que las temáticas trabajadas abordan diferentes aspectos de la sexualidad de forma más explícita, lo que contribuye a que afloren emociones de vergüenza y se muestren más retraídos al no están habituados a comentar aspectos relacionados con la sexualidad en público. De acuerdo con los resultados de otras investigaciones, estos 
hallazgos podrían ser explicados por las actitudes negativas que muchas personas con DFI han desarrollado hacia la sexualidad, algo que viene impulsado por las creencias y valores que son transmitidos por las personas de su entorno y las actitudes de estos últimos hacia la sexualidad del colectivo con DFI. En este sentido, Jahoda \& Pownall (2014) concluyeron que la actitud de las personas del entorno podría estar dificultando la discusión abierta sobre aspectos relacionados con la sexualidad. También en la investigación de Morell-Mengual et al. (2017) hallaron que los padres presentaban una tolerancia moderada-conservadora hacia la sexualidad de las personas con DFI, presentando creencias erróneas sobre la capacidad de controlar los impulsos sexuales de sus hijos/as. Así pues, los resultados de esta investigación reflejarían la necesidad de abordar de forma continuada en el tiempo el desarrollo sexual del colectivo con DFI, a fin de eliminar las barreras a las que se enfrentan, dado que una intervención puntual no será suficiente para cambiar sus falsas creencias.

\section{CONCLUSIONES}

Los resultados obtenidos nos llevan a concluir que la metodología empleada en los programas de educación sexual para personas con DFI es relevante en la medida en la que promueven en mayor o menor medida la implicación de los mismos en su proceso de aprendizaje. Así mismo, este estudio contribuye a resaltar la necesidad de transmitir los conceptos empleando estrategias adaptadas al colectivo y que les motiven y favorezcan su implicación. Derivado de todo ello, cabe resaltar, en primer lugar, la importancia de que los/las profesionales que aplican el programa integren la educación sexual como una actividad más en sus respectivos centros ocupacionales a fin de normalizar la sexualidad en este colectivo. Y, en segundo lugar, es recomendable que las personas encargadas de aplicar los programas de educación afectivo sexual en este colectivo sean aquellas que mejor conozcan al grupo y con quienes los usuarios y las usuarias tengan establecido un buen rapport, favoreciendo la apertura de los participantes a expresar sus dudas y preocupaciones sobre temas referentes a la sexualidad. Por último, cabría comentar la dificultad de comparar nuestros resultados con los obtenidos en otros estudios dada la poca literatura sobre el tema abordado, por lo que nuestros hallazgos deben tomarse con cierta cautela. Por ello, en futuras investigaciones, convendría analizarla el efecto del diseño del programa sobre la implicación de los/as usuarios/as con DFI, a fin de aportar un mayor conocimiento en este campo.

\section{REFERENCIAS}

Aceldo, M. A., Aguado, A. L., \& Arias, B. (2006). Eficacia de un programa de educación sexual en jóvenes con discapacidad intelectual. Análisis y Modificación de Conducta, 32(142), 217-239. http://rabida.uhu.es/dspace/bitstream/handle/10272/11944/Eficacia_de un_progr ama.pdf;jsessionid $=\mathrm{E} 24118 \mathrm{D} 840942 \mathrm{~F} 02 \mathrm{~F} 5873 \mathrm{CFC} 038 \mathrm{~B} 92 \mathrm{~B} 3$ ? sequence $=2$

Cosentino,S.,Maia,K.,Rodrigues,M.,Rossetto, M.\& Silva, V. (2010). Educational workshops on adolescent sexuality: School as scenario. Revista electrónica cuatrimestral de enfermería, 20(1),1-8. https://dialnet.unirioja.es/servlet/articulo?codigo $=3681316$ 
Díaz-Rodríguez, I. M., Gil-Lario, M. D., Ballester-Arnal, R., \& Molero-Mañes, R. (2014). Conocimientos, comportamiento y actitudes sexuales en adultos con discapacidad Intelectual. International Journal of Developmental and Educational Psychology INFAD Revista de Psicología, 3(1), 415-422. http://doi.org/10.17060/ijodaep.2014.n1.v3.519

Gil-Llario, M. D., Ballester-Arnal, R., Caballero-Gascón, L., \& Escalera, C. (2019). Programa SALUDIVERSEX. Programa de educación afectivo-sexual para adultos con diversidad funcional intelectual. Pirámide.

Gil-Llario, M. D., Morell-Mengual, V., Díaz-Rodríguez, I., \& Ballester-Arnal, R. (2019). Prevalence and sequelae of self-reported and other-reported sexual abuse in adults with intellectual disability. Journal of Intellectual Disability Research, 63(2), 138-148. https://doi.org/10.1111/jir.12555

Giménez-García, C., Gil-Llario, M. D., Ruiz-Palomino, E., \& Díaz Rodríguez, I. (2017). Abuso sexual y discapacidad intelectual: cómo identifican y valoran la experiencia las personas con discapacidad intelectual y los profesionales que les atienden. International Journal of Developmental and Educational Psychology. Revista INFAD de Psicología, 4(1), 129-136. https://doi.org/10.17060/ijodaep.2017.n1.v4.1035

Jahoda, A., \& Pownall, J. (2014). Sexual understanding, sources of information and social networks; the reports of young people with intellectual disabilities and their non-disabled peers. Journal of Intellectual Disability Research, 58(5), 430-441. https://doi.org/10.1111/jir.12040

Kellam, S. G., \& Langevin, D. J. (2003). A Framework for Understanding "Evidence" in Prevention Research and Programs. Prevention Science, 4(3), 137-153. https://doi.org/https://doi.org/10.1023/A:1024693321963

Morell-Mengual, V., Gil-Llario, M. D., Díaz-Rodríguez, I., \& Caballero-Gascón, L. (2017). Actitudes de padres, profesionales y población general hacia la sexualidad de las personas con discapacidad física e intelectual. International Journal of Developmental and Educational Psychology. Revista INFAD de Psicología, 4(1), 173-184. https://doi.org/10.17060/ijodaep.2017.n1.v4.1040

Navarro, Y. (2012). Percepción de la sexualidad y el amor en una muestra de personas con discapacidad intelectual. Información Psicológica, 103, 15-30. http://www.informaciopsicologica.info/OJSmottif/index.php/leonardo/article/vie $\mathrm{w} / 53 / 44$

Navarro, Y., Torrico, E., \& López, M. J. (2010). Programa de intervención psicosexual en personas con discapacidad intelectual. Educación y Diversidad, 4(2), 75-92. https://dialnet.unirioja.es/servlet/articulo?codigo $=3276492$

Roden, R. C., Schmidt, E. K., \& Holland-Hall, C. (2020). Sexual health education for adolescents and young adults with intellectual and developmental disabilities: recommendations for accessible sexual and reproductive health information. The Lancet Child \& Adolescent Health, 4(9), 699-708. https://doi.org/10.1016/S2352-4642(20)30098-5

Schaafsma, D., Stoffelen, J., Kok, G., \& Curfs, L. (2013). Exploring the Development of Existing Sex Education Programmes for People with Intellectual Disabilities: 
An Intervention Mapping Approach. Journal of Applied Research in Intellectual Disabilities, 26(2), 157-166. https://doi.org/10.1111/jar.12017

Schaalma, H. P., Abraham, C., Gillmore, M. R., \& Kok, G. (2004). Sex Education as Health Promotion: What Does It Take? Archives of Sexual Behavior, 33(3), 259-269. http://doi.org/10.1023/B:ASEB.0000026625.65171.1d

Verdugo, M. Á., Alcedo, M., Bermejo, B., \& Aguado, A. (2002). El abuso sexual en personas con discapacidad intelectual. Psicothema, 14(1), 124-129. http://www.psicothema.com/psicothema.asp?id=3482

Whitehouse, M. A., \& McCabe, M. P. (1997). Sex Education Programs for People with Intellectual Disability: How Effective Are They? Education and Training in Mental Retardation and Developmental Disabilities, 32(3), 229-240. https://www.proquest.com/docview/619150188/72C7F328079F4DDCPQ/1?acc ountid $=14777$

Whittle, C., \& Butler, C. (2018). Sexuality in the lives of people with intellectual disabilities: A meta-ethnographic synthesis of qualitative studies. Research in Developmental Disabilities,

75 , 68-81. https://doi.org/10.1016/j.ridd.2018.02.008 